\title{
Cigarette smoking is associated with higher thyroid hormone and lower TSH levels: the PREVEND study
}

\author{
Eke G. Gruppen ${ }^{1,2} \cdot$ Jenny Kootstra-Ros ${ }^{3} \cdot$ Anneke Muller Kobold $^{3} \cdot$ Margery A. Connelly $^{4}$ - Daan Touw ${ }^{5}$. \\ Jens H. J. Bos ${ }^{6}$ - Eelko Hak ${ }^{6}$. Thera P. Links ${ }^{2}$ - Stephan J. L. Bakker ${ }^{1}$ - Robin P. F. Dullaart ${ }^{2}$
}

Received: 24 September 2019 / Accepted: 26 October 2019 / Published online: 9 November 2019

(c) The Author(s) 2019

\begin{abstract}
Purpose The extent to which smoking is associated with thyroid stimulating hormone (TSH), free thyroxine (FT4), and free triiodothyronine (FT3) when taking account of clinical variables including alcohol consumption is unclear. We aimed to determine associations of TSH, FT4, and FT3 levels with current smoking.

Methods A cross-sectional study was performed in 5766 euthyroid participants (Prevention of Renal and Vascular EndStage Disease cohort). Current smoking was determined by self-report, categorized as never, former, and current $(\leq 20$ and $>20$ cigarettes per day). Smoke exposure was determined by urinary cotinine.

Results Current smoking of $\leq 20$ and $>20$ cigarettes per day was associated with lower TSH and higher FT3 levels. FT4 levels were higher in subjects smoking $<20$ cigarettes per day vs. never and former smokers. Current smokers also consumed more alcohol. Multivariable linear regression analyses adjusted for age, sex, anti-TPO autoantibody positivity, alcohol consumption, and other variables demonstrated that lower TSH, higher FT4 and higher FT3 were associated with smoking $\leq 20$ cigarettes per day vs. subjects who never smoked $(P<0.001, P=0.018$, and $P<0.001$, respectively) without a further significant incremental effect of smoking $>20$ cigarettes per day. In agreement, TSH was inversely, whereas FT4 and FT3 levels were positively associated with urinary cotinine $(P<0.001$ for each). In contrast, alcohol consumption $>30 \mathrm{~g}$ per day conferred higher TSH and lower FT3 levels.

Conclusions Cigarette smoking is associated with modestly higher FT4 and FT3, and lower TSH levels, partly opposing effects of alcohol consumption.
\end{abstract}

Keywords Alcohol consumption $\cdot$ Antiperoxidase autoantibodies $\cdot$ Cigarette smoking $\cdot$ FT4 $\cdot$ FT3 $\cdot$ TSH

Supplementary information The online version of this article (https:// doi.org/10.1007/s12020-019-02125-2) contains supplementary material, which is available to authorized users.

\section{Eke G. Gruppen}

e.g.gruppen@umcg.nl

1 Department of Nephrology, University of Groningen, University Medical Center Groningen, Groningen, The Netherlands

2 Department of Endocrinology, University of Groningen, University Medical Center Groningen, Groningen, The Netherlands

3 Department of Laboratory Medicine, University of Groningen, University Medical Center Groningen, Groningen, The Netherlands

\section{Introduction}

Disorders in thyroid function are very common in the general population [1,2], and it is increasingly appreciated that variations in thyroid function, as inferred from circulating levels of thyroid stimulating hormone (TSH), free thyroxine (FT4), and free triiodothyronine (FT3) within

4 Laboratory Corporation of America Holdings (LabCorp), Morrisville, NC, USA

5 Department of Clinical Pharmacy and Pharmacology, University of Groningen, University Medical Center Groningen, Groningen, The Netherlands

6 Clinical Pharmacoepidemiology, University of Groningen, Groningen Research Institute of Pharmacy, Unit PharmacoEpidemiology and PharmacoEconomics, Groningen, The Netherlands 
their respective reference ranges, may impact on a number of health issues including atherosclerosis susceptibility [3-6]. Although not unequivocally reported, it is also noteworthy that variations in thyroid function within the reference range could affect life expectancy [7, 8]. It is, therefore, relevant to better understand the impact of life style factors on thyroid function status.

It has long been recognized that cigarette smoking may affect thyroid function, conceivably attributable to exposure to toxic metabolites, enhanced sympathetic nervous activity, or by affecting thyroid-directed autoimmune responses $[4,9,10]$. In the eighties of the last century, it was already documented that smoking cessation elicits a rise in TSH and a decrease in T4 [11]. Indeed, with some exceptions, evidence has accumulated since that cigarette smoking is associated lower TSH and higher FT4 levels, whereas T3 and FT3 were also found to be higher or unaltered $[9,10,12,13]$. In addition, using serum or urinary cotinine as a measure of nicotine exposure it was found that smoke exposure is associated with lower TSH levels $[14,15]$.

Among other life style factors, alcohol consumption has been suggested to lower FT4 [16]. Moreover, FT4 and FT3 levels are decreased in alcohol abusers after alcohol withdrawal $[17,18]$. Given that cigarette smoking may frequently coincide with more heavy alcohol consumption $[18,19]$, it is plausible to take account of alcohol use when evaluating the association of cigarette smoking with thyroid hormone levels. However, epidemiological surveys on the effect of smoking on thyroid function parameters thus far did not comprehensively assess the extent to which such associations are independent of alcohol consumption and other relevant characteristics.

For this reason the present study was initiated to determine the association of cigarette smoking assessed by selfreport as well as by urinary cotinine concentrations with TSH, FT4, and FT3 taking account of alcohol consumption, as well as other clinical and laboratory covariates in the Prevention of Renal and Vascular End-Stage Disease (PREVEND) cohort, a large and comprehensively characterized population from the north of the Netherlands.

\section{Materials and methods}

\section{Study population}

The study was performed in the frame of the PREVEND cohort study, which investigates vascular and renal damage among predominantly white inhabitants of the city of Groningen, The Netherlands, as reported in detail elsewhere [19-23]. The study was approved by the Medical Ethics Committee of the University Medical Center Groningen and conformed to the principles outlined in the Declaration of
Helsinki. All participants gave written informed consent. Briefly, in 1997-1998, all inhabitants of the city of Groningen, aged $28-75$ years, were sent a short questionnaire on demographics and cardiovascular morbidity and a vial to collect an early morning urine sample $(n=85,421)$. Pregnant women and diabetic subjects using insulin were excluded. Altogether 40,856 subjects responded (47.8\%), and their urinary albumin concentration was determined. Subjects with a urinary albumin concentration $\geq 10 \mathrm{mg} / \mathrm{L}$ $(n=7768)$ were invited to participate, of whom 6000 were enrolled. In addition, a randomly selected group with a urinary albumin concentration $<10 \mathrm{mg} / \mathrm{L}(n=3394)$ was invited to participate in the cohort, of whom 2592 were enrolled. The initial study population was comprised of 8592 subjects who completed the total screening program. For the present study, we used data from participants who completed the second screening round (2001-2003; $n=$ 6894), excluding those with missing values for smoking behavior, thyroid function tests, as well as those who were not euthyroid (see Measurements and Definitions) and those using thyroid hormones, antithyroid drugs, amiodarone, or lithium carbonate. This left a cohort of 5766 participants with sufficient information for analysis (Fig. 1).

\section{Measurements and definitions}

Euthyroidism was defined as TSH, FT4, and FT3 levels all within the euthyroid reference range (TSH: $0.27-4.20 \mathrm{mU} / \mathrm{L}$; FT4: $12-22 \mathrm{pmol} / \mathrm{L}$; FT3:3.1-6.8 pmol/L). Cigarette smoking was estimated by questionnaire, and was categorized as (i) never; (ii) former; (iii) current $\leq 20$ cigarettes per day, and (IV) current $>20$ cigarettes per day. In addition, urinary cotinine concentrations were measured. Former smokers were subjects who were nonsmokers at the time of the second screening round but had ever smoked in their life; current smokers were those who reported smoking at this screening. Alcohol intake was estimated by using predefined categories covering a time period of one month [19, 22]. Alcohol intake was then calculated as alcoholic drinks per day with one drink being considered to be equivalent to $10 \mathrm{~g}$ of alcohol, regardless of the type of beverage [19]. Alcohol intake was categorized in (i) no/rarely; (ii) $0.1-10 \mathrm{~g}$ per day (occasional to light); (iii) $10-30 \mathrm{~g}$ per day (moderate), and (IV) $>30$ g per day (heavy).

The presence of a self-reported history of myocardial infarction, percutaneous coronary intervention, coronary artery bypass surgery, stroke, or the diagnosis of narrowing of one or both carotid arteries was defined as cardiovascular disease. Type 2 diabetes mellitus was defined as a fasting serum glucose concentration $>7.0 \mathrm{mmol} / \mathrm{L}$, a nonfasting serum glucose concentration $>11.1 \mathrm{mmol} / \mathrm{L}$, a self-report of a physician diagnosis, or the use of glucose-lowering drugs [23]. 
Fig. 1 Flowchart depicting PREVEND participants included or excluded for the purposes of this study

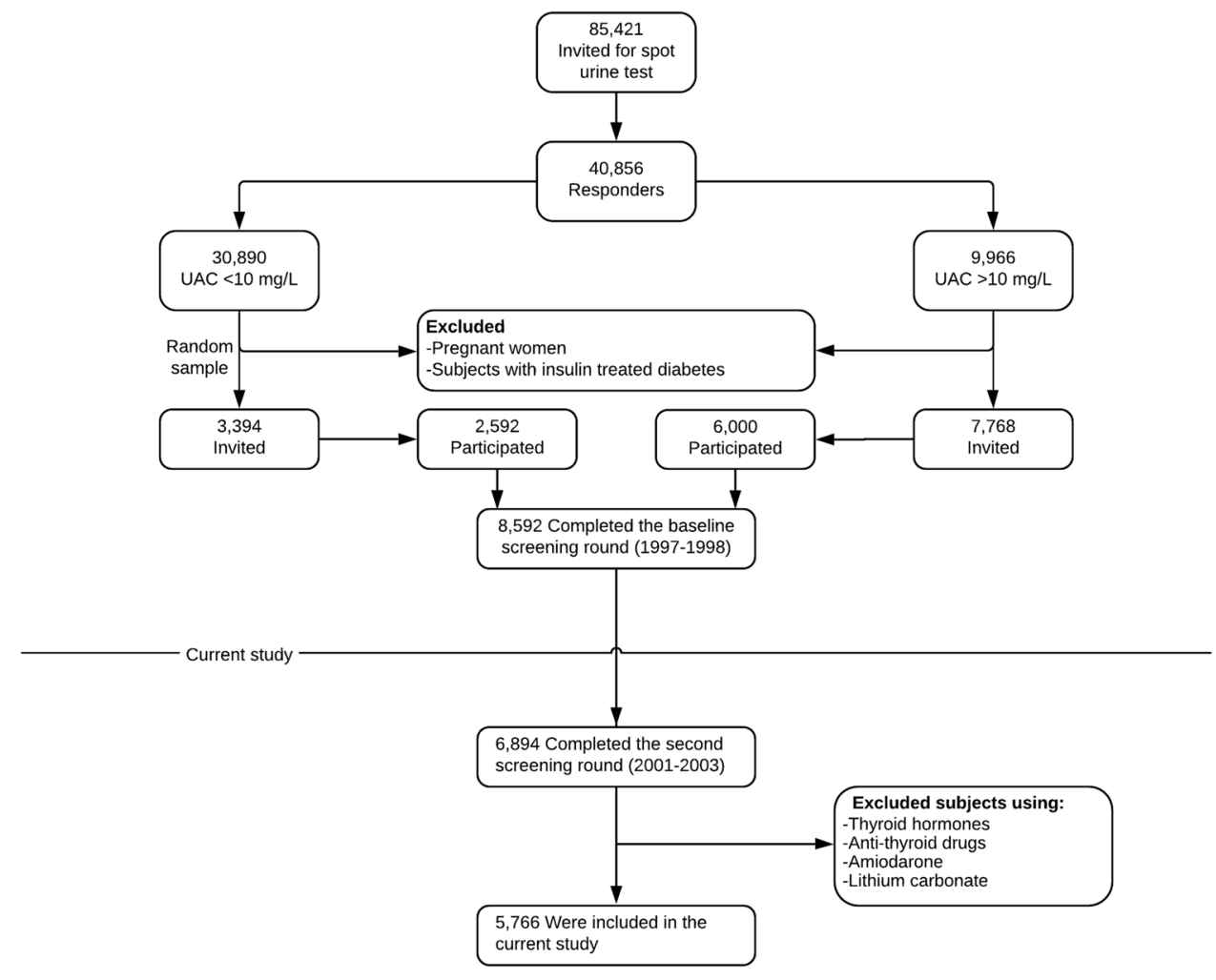

Information on medication use was combined with information from IADB.nl, a data base containing information of prescribed medication in public pharmacies in The Netherlands (http://www.iadb.nl/) [24]. Body mass index (BMI) was calculated as weight $(\mathrm{kg})$ divided by height squared (m). Blood pressure was measured using an automatic device (Dinamap XL model 9300 series; Johnson-Johnson Medical, Tampa, FL, USA). Urinary albumin excretion (UAE) was measured in two consecutive $24 \mathrm{~h}$ urine collections after oral and written instruction. The participants were asked to avoid heavy exercise as much as possible during the urine collection. Subjects were also instructed to postpone their urine collection in case of urinary tract infection, menstruation, or fever. The UAE results were averaged for analysis. Estimated glomerular filtration rate (eGFR) was calculated applying the combined creatinine cystatin C-based Chronic Kidney Disease Epidemiology Collaboration equation [25]. The participants were instructed to have their blood samples being taken in the morning after an overnight fasting period.

\section{Laboratory methods}

Venous blood samples were obtained after $15 \mathrm{~min}$ rest. Venous plasma and serum samples were obtained by centrifugation at $1400 \times g$ for $15 \mathrm{~min}$ at $4{ }^{\circ} \mathrm{C}$. Serum glucose was measured shortly after blood sampling. Ethylenediaminetetraacetic acid anticoagulated plasma and heparinized plasma and serum was obtained by centrifugation at $4{ }^{\circ} \mathrm{C}$, and stored at $-80{ }^{\circ} \mathrm{C}$ until analysis. Cotinine concentrations were measured with with Enzyme Multiplied Immunoassay Technique on the Abbott Architect c8000 system (Abbott Laboratories, Abbott Park, IL, USA). Serum TSH, FT4, FT3, and antithyroid peroxidase (anti-TPO) autoantibodies were determined using the Roche Modular E170 Analyzer electrochemiluminescent immunoassays (Roche Diagnostics, Mannheim, Germany). Anti-TPO autoantibodies were considered positive using a cut-off value as indicated by the supplier $(\geq 35 \mathrm{kU} / \mathrm{L})$. Twenty-four hour urinary cotinine levels were measured with Enzyme Multiplied Immunoassay Technique on the Abbott Architect c8000 system (Abbott Laboratories, Abbott Park, IL).

Total cholesterol was measured on an automatic analyzer (MEGA; Merck, Darmstadt, Germany) using the CHOD-PAP-method. Glucose was measured using Kodak Ektachem dry chemistry (Eastman Kodak, Rochester, New York, USA). HDL cholesterol and triglycerides were determined using the nuclear magnetic resonance (NMR)derived LP4 lipoprotein profile deconvolution algorithm as described [26]. To this end NMR spectra were acquired on a Vantera ${ }^{\circledast}$ Clinical Analyzer at LabCorp (Morrisville, NC, USA). Serum creatinine was measured by an enzymatic method on a RocheModular analyzer (Roche Diagnostics, Mannheim, Germany). Serum cystatin C was measured by Gentian Cystatin C Immunoassay (Gentian AS, Moss, Norway) on a Modular analyzer (Roche Diagnostics). 
Urinary albumin was measured by nephelometry (Dade Behring Diagnostic, Marburg, Germany) [19].

\section{Statistical analyses}

Data analysis was performed using IBM SPSS software (version 23.0, SPSS Inc., USA). Normally distributed data are given as mean \pm SD and nonparametrically distributed data are presented as median (interquartile range). Categorical variables are given as numbers and \%. Anti-TPO autoantibody titers (as continuous variable), TSH, triglycerides, urine cotinine, and UAE were natural logarithm $\left(\log _{e}\right)$ transformed in order to achieve approximately normal distributions. Differences in continuous variables between categories of cigarette smoking were determined by Analysis of Variance with subsequent Bonferroni procedure to correct for multiple comparisons. Differences in TSH, FT4, and FT3 according to anti-TPO antibody status were determined by unpaired $T$-tests. Proportions of dichotomous variables across categories of cigarette smoking were determined by $\chi^{2}$ tests. Pearson correlation coefficients were calculated to determine univariate relationships. Multivariable linear regression analyses were used to discern the independent association of current cigarette smoking with TSH, FT4, and FT3. In these analyses, the category "never smoked" was used as the reference category. In these analyses, we also used categorized alcohol consumption as variable with the category "no/rarely" as reference. In addition, analyses were repeated with urinary cotinine data instead of self-reported smoking status. In these analyses, urinary cotinine concentrations of 0 were set at 0.01 to allow for $\log _{\mathrm{e}}$ transformation. Standardized regression coefficients $(\beta)$ were used to discern the strength of associations of current smoking ( $\leq$ and $>20$ cigarettes per day) with TSH, FT4, and FT3. Two-sided $P$-values $<0.05$ were considered significant.

\section{Results}

The clinical and laboratory characteristics of the participants are shown in Table 1. Of the 5766 participants, $29.0 \%$ had never smoked, $42.2 \%$ were former smokers, $24.9 \%$ smoked $\leq 20$ cigarettes per day, and $3.9 \%$ smoked $>20$ cigarettes per day. Urinary cotinine concentrations, measured in 5722 subjects, were highest in subjects who smoked $>20$ cigarettes per day, and virtually absent in never smokers and former smokers. Current smokers were younger than former smokers and more likely to be men. There were also differences in hemodynamics across the four groups, with systolic blood pressure being lower in current smokers vs. former smokers and pulse rate being highest in subjects who smoked $>20$ cigarettes per day. The most frequent use of antihypertensive and lipid lowering drug was observed in the group of former smokers. Alcohol consumption was lowest in subjects who never smoked and highest in subjects who smoked $>20$ cigarettes per day. eGFR was lowest and UAE was highest in former smokers. Total cholesterol was highest and HDL cholesterol was lowest in subjects who smoked $\leq 20$ cigarettes per day, whereas triglycerides were lowest in subjects who smoked $>20$ cigarettes per day. As shown in Table 1, TSH levels were lower in both groups of smokers vs. never and former smokers. FT4 levels were higher in subjects who smoked $\leq 20$ cigarettes per day vs. never and former smokers, whereas FT3 levels were highest in both groups of smokers vs. never and former smokers. Anti-TPO antibody titers were highest in current smokers. The highest prevalence of positive anti-TPO antibodies was observed in subjects who smoked $>20$ cigarettes per day.

TSH was higher, whereas FT4 and FT3 levels were lower in women vs. men (women $(n=2768)$ : TSH 1.65 (1.14-2.32) $\mathrm{mU} / \mathrm{L}$, FT4 $15.5 \pm 1.8 \mathrm{pmol} / \mathrm{L}$, and FT3 $4.7 \pm$ $0.5 \mathrm{pmol} / \mathrm{L}$ vs. men $(n=3008)$ : TSH $1.50(1.08-2.08) \mathrm{mU} / \mathrm{L}$, FT4 $16.0 \pm 1.9 \mathrm{pmol} / \mathrm{L}$, and FT3 $4.7 \pm 0.5 \mathrm{pmol} / \mathrm{L}, P<0.001$ for each comparison between women and men). Positive antiTPO antibodies were more frequently present in women vs. men (Supplemental Table 1). Moreover, when thyroid function was assessed according to positive anti-TPO antibody status, TSH was found to be higher, whereas FT4 and FT3 were lower in subjects who were positive for anti-TPO antibodies (Supplemental Table 1).

Multivariable linear regression analyses demonstrated that TSH levels were associated inversely with current cigarette smoking independent of age and sex with the never smokers as reference category (Table 2; model 1). Conversely, there were positive associations of FT4 (Table 2, model 2) and FT3 (Table 2, model 3) with current cigarette smoking. However, as indicated by the standardized regression coefficients $(\beta)$, these associations were not stronger in subjects who smoked $>20$ cigarettes per day vs. subjects who smoked $\leq 20$ cigarettes per day. We also performed analysis with urinary cotinine concentrations as objective reflection of smoke exposure (Table 3). The univariate inverse correlations of TSH with urinary cotinine are shown in Fig. 2a, whereas the positive correlations with FT4 and FT3 are shown in Fig. 2b, c, respectively.

Given many differences in clinical and laboratory variables across the four categories of smoking behavior (Table 1), we next performed multivariable linear regression analyses in which we took account of a large number of relevant clinical and laboratory variables. As shown in Table 4, these analyses also showed that TSH levels remained inversely, whereas FT4 and FT3 levels remained associated positively with current smoking. These analyses also did not demonstrate stronger associations in subjects 
Table 1 Clinical and laboratory characteristics according to smoking status in 5766 participants of the PREVEND study

\begin{tabular}{|c|c|c|c|c|c|}
\hline & Never smokers & Former smokers & $\begin{array}{l}\text { Current smokers } \leq 20 \\
\text { cigarettes per day }\end{array}$ & $\begin{array}{l}\text { Current smokers }>20 \\
\text { cigarettes per day }\end{array}$ & $P$-value \\
\hline Urine cotinine $(\mathrm{ng} / \mathrm{mL})^{*}$ & $0[0-0]$ & $0[0-0]$ & $1191[660-1721]$ & 1744 [1310-2296] & $<0.001$ \\
\hline Number $(\%)$ & $1670(29.0)$ & $2436(42.2)$ & $1433(24.9)$ & $227(3.9)$ & \\
\hline Age (years) & $51 \pm 12$ & $56 \pm 12$ & $52 \pm 11^{\mathrm{d}}$ & $50 \pm 8^{\mathrm{d}}$ & $<0.001$ \\
\hline $\operatorname{Sex}(\mathrm{M}: n ; \%)$ & $750(44.9)$ & $1408(57.8)$ & $728(50.8)$ & $112(49.3)$ & $<0.001$ \\
\hline Systolic blood pressure (mm $\mathrm{Hg}$ ) & $125 \pm 8$ & $129 \pm 19$ & $123 \pm 18^{\mathrm{d}}$ & $124 \pm 16^{\mathrm{e}}$ & $<0.001$ \\
\hline Diastolic blood pressure (mm $\mathrm{Hg}$ ) & $72 \pm 9$ & $75 \pm 9$ & $73 \pm 9^{\mathrm{d}}$ & $75 \pm 8^{\mathrm{b}}$ & $<0.001$ \\
\hline Pulse pressure (mm Hg) & $53 \pm 13$ & $54 \pm 14$ & $51 \pm 11^{\mathrm{a}, \mathrm{d}}$ & $53 \pm 12^{\mathrm{b}, \mathrm{d}}$ & $<0.001$ \\
\hline Pulse rate (beats/min) & $68 \pm 10$ & $68 \pm 10$ & $69 \pm 10^{\mathrm{c}, \mathrm{d}}$ & $74 \pm 10^{\mathrm{a}, \mathrm{d}}$ & $<0.001$ \\
\hline BMI $\left(\mathrm{kg} / \mathrm{m}^{2}\right)$ & $26.5 \pm 4.4$ & $27.2 \pm 4.3$ & $27.2 \pm 4.3^{\mathrm{a}}$ & $26.5 \pm 4.5$ & $<0.001$ \\
\hline Diabetes $(n ; \%)$ & $91(5.4)$ & $167(6.9)$ & $69(4.8)$ & $12(5.3)$ & 0.050 \\
\hline History of CVD $(n ; \%)$ & $46(2.8)$ & $211(8.7)$ & $106(7.4)$ & $8(3.5)$ & $<0.001$ \\
\hline Antihypertensive drugs $(n ; \%)$ & $274(16.4)$ & $642(26.4)$ & $236(16.5)$ & $39(17.2)$ & $<0.001$ \\
\hline Glucose lowering drugs $(n ; \%)$ & $54(3.2)$ & $94(3.9)$ & $39(2.7)$ & $3(1.3)$ & 0.082 \\
\hline Lipid lowering drugs $(n ; \%)$ & $102(6.1)$ & $267(11.0)$ & $140(9.8)$ & $22(9.7)$ & $<0.001$ \\
\hline Alcohol consumption (g/day) & & & & & $<0.001$ \\
\hline $0 /$ rarely $(n ; \%)$ & $515(30.8)$ & $503(20.6)$ & $333(23.2)$ & $54(23.8)$ & \\
\hline $0.1-10(n ; \%)$ & $352(21.1)$ & $377(15.5)$ & $219(15.3)$ & $14(6.2)$ & \\
\hline $10-30(n ; \%)$ & $557(33.4)$ & $778(31.9)$ & $458(32.0)$ & $56(24.7)$ & \\
\hline$>30(n ; \%)$ & $246(14.7)$ & $778(31.9)$ & $423(29.5)$ & $103(45.3)$ & \\
\hline Glucose (mmol/L) & $5.0 \pm 1.2$ & $5.1 \pm 1.2$ & $4.9 \pm 1.1^{\mathrm{d}}$ & $5.0 \pm 1.2$ & \\
\hline eGFR (ml/min/1.73 $\left.\mathrm{m}^{2}\right)$ & $95 \pm 16$ & $91 \pm 18$ & $91 \pm 18^{\mathrm{a}}$ & $97 \pm 14^{\mathrm{d}}$ & $<0.001$ \\
\hline $\mathrm{UAE}(\mathrm{mg} / 24 \mathrm{~h})$ & $7.8(5.8-2.8)$ & $9.2(6.2-18.1)$ & $8.8(6.015 .9)^{\mathrm{a}, \mathrm{f}}$ & $7.8(5.8-12.8)^{\mathrm{a}}$ & $<0.001$ \\
\hline Total cholesterol (mmol/L) & $5.3 \pm 1.0$ & $5.4 \pm 1.0$ & $5.5 \pm 1.0^{\mathrm{a}}$ & $5.3 \pm 1.0^{\mathrm{a}, \mathrm{f}}$ & $<0.001$ \\
\hline HDL cholesterol $(\mathrm{mmol} / \mathrm{L})$ & $1.3 \pm 0.3$ & $1.3 \pm 0.3^{\mathrm{b}}$ & $1.2 \pm 0.3^{\mathrm{a}, \mathrm{e}}$ & $1.3 \pm 0.3^{\mathrm{b}, \mathrm{e}}$ & $<0.001$ \\
\hline Triglycerides (mmol/L) & $1.3(0.7-1.4)$ & $1.1(0.8-1.6)$ & $1.2(0.9-1.7)^{\mathrm{b}}$ & $1.0(0.7-1.4)^{\mathrm{b}}$ & $<0.001$ \\
\hline TSH (mU/L) & $1.68(1.17-2.39)$ & $1.62(1.14-2.21)$ & $1.44(1.00-1.96)^{\mathrm{a}, \mathrm{d}}$ & $1.48(0.94-1.91)^{\mathrm{a}, \mathrm{d}}$ & $<0.001$ \\
\hline FT4 (pmol/L) & $15.7 \pm 1.9$ & $15.7 \pm 1.9$ & $15.9 \pm 1.9^{\mathrm{c}, \mathrm{d}}$ & $15.9 \pm 2.0$ & $<0.001$ \\
\hline FT3 (pmol/L) & $4.9 \pm 0.5$ & $4.8 \pm 0.5$ & $4.9 \pm 0.5^{\mathrm{a}, \mathrm{d}}$ & $5.0 \pm 0.5^{\mathrm{a}, \mathrm{d}}$ & $<0.001$ \\
\hline Anti-TPO autoantibody titer (IU/mL) & $9.8(7.8-13.2)$ & $9.6(7.6-12.9)$ & $11.4(9.3-15.0)^{\mathrm{a}, \mathrm{d}}$ & $12.7(10.7-16.3)^{\mathrm{a}, \mathrm{d}}$ & $<0.001$ \\
\hline Anti-TPO autoantibody positive $(n ; \%)$ & $150(8.9)$ & $163(6.7)$ & $110(7.7)$ & $22(9.7)$ & 0.036 \\
\hline
\end{tabular}

Smoking status is categorized as never; former and current, which is divided in $<20$ and $\geq 20$ cigarettes per day. Alcohol consumption is categorized in 0/rarely; $0.10 ; 10-30$ and $>30$ g per day. Ten grams of alcohol is equivalent to one alcoholic drink. Anti-TPO autoantibodies were not available four subjects. Data are given in mean $\pm \mathrm{SD}$, medians (interquartile ranges), or in numbers (\%). Between group differences are determined by analysis of variance. Bonferroni method is applied to correct for multiple comparisons. $P$-value: $P$-value by analysis of variance or by $\chi^{2}$-analysis. anti-TPO antithyroid peroxidase, BMI body mass index, $C V D$ cardiovascular disease, eGFR estimated glomerular filtration rate, FT4 free thyroxine, FT3 freetriiodothyronine, $H D L$ high density lipoproteins, TSH thyroid stimulating hormone, UAE urinary albumin excretion

Asterisk indicates that urine cotinine was measured in 5722 participants

${ }^{\mathrm{a}} P<0.001$

${ }^{\mathrm{b}} P<0.01$

${ }^{\mathrm{c}} P<0.05$ vs. never smokers

${ }^{\mathrm{d}} P<0.001$

${ }^{\mathrm{e}} P<0.01$

${ }^{\mathrm{f}} P<0.05$ vs. former smokers

who smoked $>20$ cigarettes per day vs. subjects who smoked $\leq 20$ cigarettes per day. Notably, these analyses also demonstrated lower TSH, higher FT4, and higher FT3 levels in men, independent of anti-TPO antibody status, as well as inverse associations of TSH, FT4, and FT3 with age. FT4 was positively and FT3 was inversely associated with BMI. Of further note, TSH levels were positively, whereas FT3 levels were inversely associated with heavy alcohol 
Table 2 Multivariable linear regression analyses demonstrating associations of thyroid function variables with cigarette smoking adjusted for age and sex in 5766 participants of the PREVEND study

\begin{tabular}{|c|c|c|c|c|c|c|}
\hline & \multicolumn{2}{|c|}{ Model 1: TSH } & \multicolumn{2}{|c|}{ Model 2: FT4 } & \multicolumn{2}{|c|}{ Model 3: FT3 } \\
\hline & $\beta$ & $P$-value & $\beta$ & $P$-value & $\beta$ & $P$-value \\
\hline Age & -0.021 & $<0.001$ & -0.028 & 0.036 & -0.167 & $<0.001$ \\
\hline Sex (M vs. F) & -0.075 & $<0.001$ & 0.117 & $<0.001$ & 0.286 & $<0.001$ \\
\hline \multicolumn{7}{|l|}{ Smoking } \\
\hline Never (reference) former & -0.037 & 0.020 & 0.026 & 0.10 & -0.008 & 0.59 \\
\hline $\begin{array}{l}\text { Current } \\
\leq 20 \text { cigarettes per day }\end{array}$ & -0.140 & $<0.001$ & 0.043 & 0.006 & 0.055 & $<0.001$ \\
\hline $\begin{array}{l}\text { Current } \\
>20 \text { cigarettes per day }\end{array}$ & -0.083 & $<0.001$ & 0.018 & 0.18 & 0.058 & $<0.001$ \\
\hline
\end{tabular}

Smoking status is categorized as never (reference category); former and current, which is divided in <20 and $\geq 20$ cigarettes per day. $\beta$ : standardized regression coefficients

$F$ female, FT4 free thyroxine, FT3 free triiodothyronine, $M$ male, TSH thyroid stimulating hormone. TSH is $\log _{\mathrm{e}}$ transformed

Table 3 Multivariable linear regression analyses demonstrating associations of thyroid function variables with cotinine levels adjusted for age and sex in 5722 participants of the PREVEND study

\begin{tabular}{|c|c|c|c|c|c|c|}
\hline & \multicolumn{2}{|c|}{ Model 1: TSH } & \multicolumn{2}{|c|}{ Model 2: FT4 } & \multicolumn{2}{|c|}{ Model 3: FT3 } \\
\hline & $\beta$ & $P$-value & $\beta$ & $P$-value & $\beta$ & $P$-value \\
\hline Age & -0.007 & 0.56 & -0.033 & 0.007 & -0.137 & $<0.001$ \\
\hline $\begin{array}{l}\text { Sex } \\
\text { (M vs. F) }\end{array}$ & 0.068 & $<0.001$ & -0.111 & $<0.001$ & -0.207 & $<0.001$ \\
\hline $\begin{array}{l}\text { Urinary } \\
\text { cotinine }\end{array}$ & -0.085 & $<0.001$ & 0.038 & 0.002 & 0.045 & $<0.001$ \\
\hline
\end{tabular}

$\beta$ : standardized regression coefficients. TSH and urinary cotinine are $\log _{\mathrm{e}}$ transformed

$F$ female, FT4 free thyroxine, FT3 free triiodothyronine, $M$ male, TSH thyroid stimulating hormone

consumption. Table 5 shows the multivariable adjusted results of thyroid function with urine cotinine concentrations with an inverse association with TSH and positive associations with FT4 and FT3, comparable to the results with self-reported smoking status.

\section{Discussion}

In this large cohort of predominantly white European subjects selected for strict euthyroidism, we documented that current cigarette smoking is associated with modestly lower TSH and higher FT3 levels compared with never and former smokers. In univariate analyses, FT4 levels were higher in subjects who smoked $\leq 20$ cigarettes per day vs. never and former smokers. Multivariable linear regression analyses confirmed these findings by demonstrating that TSH levels were lower, whereas FT3 levels were higher in current smokers even independent of a comprehensive number of clinical and laboratory variables including alcohol consumption. Likewise in these multivariable analyses, FT4 levels were higher in current smokers vs. never smokers. Notably, we, did not observe an incremental effect of more heavy smoking on these thyroid function parameters as determined by self-report, as judged from the standardized regression coefficients with the never smokers as reference category. In addition, our study demonstrates an inverse relationship of TSH and positive relationships with FT4 and FT3 with urinary cotinine both in univariate and in multivariable analysis. While many earlier studies have revealed that smoking is likely to affect thyroid function status in the same direction as presently shown [9-15, 27, 28], the current report represents to our knowledge the largest survey to demonstrate that these associations are independent of alcohol consumption with confirmation by urinary cotinine concentrations as objective measure of smoke exposure.

It has been suggested that smoking may influence sympathetic nervous activity and affect thyroid-directed autoimmune responses $[4,9,10]$. In the current study, pulse rate was elevated in both categories of current smokers, but systolic blood pressure and pulse pressure were not elevated. Thus, while sympathetic nervous activity is probably to some extent enhanced in smokers [29], it remains uncertain whether this would influence thyroid hormone levels. With respect to anti-TPO autoantibodies: when antibody titers were assessed as a continuous variable, higher titers were observed in smokers who smoked $\leq 20$ and $>20$ cigarettes per day vs. never and former smokers. Further, the highest prevalence of positive anti-TPO autoantibodies was found in subjects who smoked $>20$ cigarettes per day. These findings would be consistent with an effect of smoking on thyroid-directed autoimmunity as for example widely appreciated in Graves disease and thyroidassociated ophthalmopathy $[9,10]$. In comparison, previous cohort studies have variably reported increased [15] and decreased [30, 31] anti-TPO positivity in relation to 

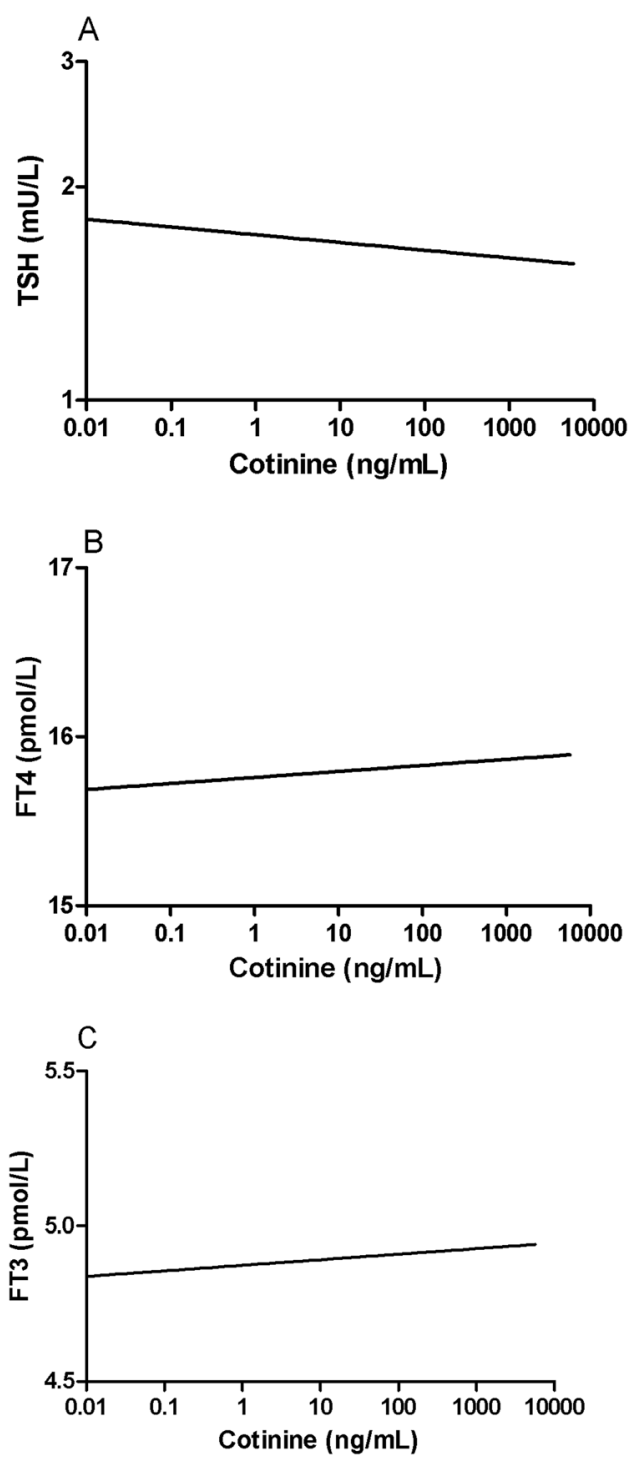

Fig. 2 Relationship of thyroid stimulating hormone (TSH) a, free thyroxine (FT4) $\mathbf{b}$, and free triiodothyronine $\mathbf{c}$ with urinary cotinine excretion in 5722 participants of the PREVEND study. TSH and urinary cotinine are $\log _{\mathrm{e}}$ transformed. Univariate correlation coefficients: TSH with urinary cotinine: $r=-0.118, P<0.001$; FT4 with urinary cotinine: $r=0.044, P=0.001$; FT3 with urinary cotinine: $r=0.079, P<0.001$

smoking behavior. In this regard it is also remarkable that even under euthyroid conditions, subjects who were positive for anti-TPO autoantibodies had higher TSH, lower FT4 and also lower FT3 levels compared with anti-TPO autoantibody negative participants.

Partly opposite effects of alcohol consumption on thyroid function parameters compared with cigarette smoking were found, with higher TSH levels in subjects who reported regular alcohol consumption, and lower FT3 levels in heavy alcohol users. Hence, given that cigarette smoking may coincide with more heavy alcohol use [18, 19], the present results reinforce our assumption that it is relevant to take account of alcohol consumption when evaluating the association of smoking with thyroid function parameters. Likewise, smoking and alcohol use also affects HDL cholesterol levels in opposite directions [19, 32]. Although earlier smaller-sized studies have suggested some effects of alcohol use on thyroid function $[16,17]$, we are not aware of large-scale population studies on the association of alcohol consumption with thyroid function parameters. Notably, however, we could not take account of other life style factors such as exercise and nutrient intake, which my coincide with alcohol consumption.

In the interpretation of the current findings it should be considered that we defined euthyroidism by TSH, FT4 as well as FT3 levels each within the reference range making use of well-established electrochemiluminescent immunoassay systems. Our selection of euthyroid subjects based on an FT3 value within the reference range, differs from other cohort studies in which FT3 measurements were not available [7, 33]. In multivariable analysis, we observed an inverse association of TSH with age in agreement with other reports from the Netherlands [2, 34]. Of note, we also observed inverse associations of FT4 with age contrasting these earlier findings [34] and even more so with respect to FT3. These relationships were interpreted to be at least in part attributable to increasing anti-TPO positivity with age [1, 34]. The higher TSH, lower FT4 and FT3 levels in women could be ascribed to female predisposition for antiTPO autoantibodies, although these associations were still present after adjustment for anti-TPO positively. Iodine intake may affect relationships of thyroid function parameters with age and sex [35]. In this regard it is relevant that the North of the Netherlands represents an geographic area of sufficient iodine intake, and was not identified as an area with insufficient or borderline iodine intake in the past [36].

Several other methodological aspects of our study should be acknowledged. We consider the comprehensive documentation of clinical and laboratory variables including urinary cotinine, which is considered to be the gold standard measure of cigarette smoke exposure, in a large cohort including participants with a wide age range as well as comparable participation of both sexes as a strength of our study. On the other hand, the number of participants with nonwhite ethnicity was low, hampering extrapolation to other ethnicities. Further, we carried out a cross-sectional study making that causal relationships could not be established. Nonetheless, that tobacco exposure is probably directly implicated in affecting thyroid function stems from one of the first reports on this issue which demonstrated a rise in TSH and a drop in T4 after smoking cessation [11]. Self-report on smoking behavior may result in inaccuracies consequent to smoking denial or difficulty in recalling the quantity of cigarettes smoked and exposure period [37], although good agreement with cotinine measurement was found in a previous report [38]. Such limitations could in 
Table 4 Multivariable linear regression analyses demonstrating independent associations of thyroid function variables with cigarette smoking in 5766 participants of the PREVEND study
Table 5 Multivariable linear regression analyses demonstrating independent associations of thyroid function variables with urinary cotinine concentration in 5722 participants of the PREVEND study

\begin{tabular}{|c|c|c|c|c|c|c|}
\hline & \multicolumn{2}{|c|}{ Model 1: TSH } & \multicolumn{2}{|c|}{ Model 2: FT4 } & \multicolumn{2}{|c|}{ Model 3: FT3 } \\
\hline & $\beta$ & $P$-value & $\beta$ & $P$-value & $\beta$ & $P$-value \\
\hline Age & -0.087 & $<0.001$ & -0.098 & $<0.001$ & -0.221 & $<0.001$ \\
\hline $\operatorname{Sex}(M$ vs. F) & -0.051 & 0.001 & 0.126 & $<0.001$ & 0.259 & $<0.001$ \\
\hline \multicolumn{7}{|l|}{ Current smoking } \\
\hline Never (reference) former & -0.027 & 0.10 & -0.037 & 0.026 & 0.003 & 0.87 \\
\hline Current $\leq 20$ cigarettes per day & -0.134 & $<0.001$ & 0.039 & 0.018 & 0.081 & $<0.001$ \\
\hline Current $>20$ cigarettes per day & -0.083 & $<0.001$ & 0.025 & 0.076 & 0.068 & $<0.001$ \\
\hline \multicolumn{7}{|l|}{ Alcohol consumption (g/day) } \\
\hline $\begin{array}{l}\text { 0/rarely (reference) } \\
0.1-10\end{array}$ & 0.032 & 0.048 & 0.035 & 0.030 & 0.012 & 0.45 \\
\hline $10-30$ & 0.063 & $<0.001$ & 0.013 & 0.46 & -0.013 & 0.44 \\
\hline$>30$ & 0.043 & 0.015 & 0.004 & 0.81 & -0.045 & 0.009 \\
\hline Positive anti-TPO autoantibodies (yes/no) & 0.134 & $<0.001$ & -0.043 & 0.002 & -0.024 & 0.059 \\
\hline
\end{tabular}

Alcohol consumption is categorized in 0/rarely (reference category); $0.10 ; 10-30$ and $>30 \mathrm{~g}$ per day. $\beta$ : standardized regression coefficients. All models were adjusted for BMI, diabetes, history of CVD, antihypertensive drugs, glucose-lowering drugs, lipid-lowering drugs, eGFR, UAE, total cholesterol, HDL cholesterol, triglycerides. UAE, urinary albumin excretion. TSH, triglycerides and UAE are $\log _{\mathrm{e}}$ transformed anti-TPO antithyroid peroxidase, $F$ female, FT4 free thyroxine, FT3 free triiodothyronine, $M$ male, TSH thyroid stimulating hormone

\begin{tabular}{|c|c|c|c|c|c|c|}
\hline & \multicolumn{2}{|c|}{ Model 1: TSH } & \multicolumn{2}{|c|}{ Model 2: FT4 } & \multicolumn{2}{|c|}{ Model 3: FT3 } \\
\hline & $\beta$ & $P$-value & $\beta$ & $P$-value & $\beta$ & $P$-value \\
\hline Age & -0.086 & $<0.001$ & -0.106 & $<0.001$ & -0.224 & $<0.001$ \\
\hline $\operatorname{Sex}(\mathrm{M}$ vs. F) & 0.053 & 0.001 & -0.125 & $<0.001$ & -0.258 & $<0.001$ \\
\hline Urinary cotinine & -0.122 & $<0.001$ & 0.044 & 0.002 & 0.091 & $<0.001$ \\
\hline \multicolumn{7}{|l|}{ Alcohol consumption (g/day) } \\
\hline $\begin{array}{l}0 / \text { rarely (reference) } \\
0.1-10\end{array}$ & 0.036 & 0.026 & 0.033 & 0.040 & 0.009 & 0.56 \\
\hline $10-30$ & 0.064 & $<0.001$ & 0.009 & 0.62 & -0.015 & 0.37 \\
\hline$>30$ & 0.040 & 0.023 & -0.001 & 0.96 & -0.044 & 0.010 \\
\hline Positive anti-TPO autoantibodies (yes/no) & 0.134 & $<0.001$ & -0.043 & 0.002 & -0.028 & 0.031 \\
\hline
\end{tabular}

Alcohol consumption is categorized in 0/rarely (reference category); 0.10; 10-30 and $>30 \mathrm{~g}$ per day. Urinary cotinine data was measured in 5722 subjects. $\beta$ : standardized regression coefficients. All models were adjusted for BMI, diabetes, history of CVD, antihypertensive drugs, glucose-lowering drugs, lipid-lowering drugs, eGFR, UAE, total cholesterol, HDL cholesterol, triglycerides. Cotinine, TSH, triglycerides and UAE are $\log _{\mathrm{e}}$ transformed

anti-TPO antithyroid peroxidase, $F$ female, FT4 free thyroxine, FT3 free triiodothyronine, $M$ male, TSH thyroid stimulating hormone part be responsible for the fact that we did not observe incremental effects of more heavy smoking as assessed by questionnaire on TSH, FT4, and FT3. In fact our data suggests linear relationships of smoke exposure with TSH (inverse) as well as with FT4 and FT3 (positive).

In conclusion, this large cross-sectional study among euthyroid predominantly white north European subjects demonstrates that cigarette smoking is associated with modest alterations in thyroid function as evidenced from lower TSH, higher FT4, and higher FT3 levels. These associations remain when taking account of clinical and laboratory variables including partly opposing effects of alcohol consumption.

\section{Data availability}

The data that support the findings of this study are available from the corresponding author upon reasonable request. 
Acknowledgements The cotinine measurement for this research was supported by a grant from the EU Joint Programme Initiative A Healthy Diet for a Healthy Life (JPI HDHL), the Food Biomarker Alliance (FOODBALL).

Authors' contributions The authors' responsibilities were as follows: E.H. and J.H.J.B. provided data of medication use. J.K.-R and A.M.K. supervised the thyroid function tests and anti-TPO measurements. D.T. supervised the cotinine measurements. M.A.C. provided lipoprotein and lipid measurements. E.G.G. and R.P.F.D. analyzed data/performed statistical analysis; E.G.G., S.J.L.B., and R.P.F.D. interpreted the data; E.G.G. and R.P.F.D. wrote the paper; E.G.G. and R.P.F.D. had primary responsibility for final content. All authors read and approved the final paper.

\section{Compliance with ethical standards}

Conflict of interest The authors declare that they have no conflict of interest.

Publisher's note Springer Nature remains neutral with regard to jurisdictional claims in published maps and institutional affiliations.

Open Access This article is distributed under the terms of the Creative Commons Attribution 4.0 International License (http://crea tivecommons.org/licenses/by/4.0/), which permits unrestricted use, distribution, and reproduction in any medium, provided you give appropriate credit to the original author(s) and the source, provide a link to the Creative Commons license, and indicate if changes were made.

\section{References}

1. J.G. Hollowell, N.W. Staehling, W.D. Flanders, W.H. Hannon, E. W. Gunter, C.A. Spencer, L.E. Braverman, Serum TSH, T4, and thyroid antibodies in the United States population (1988 to 1994): National Health and Nutrition Examination Survey (NHANES III). J. Clin. Endocrinol. Metab. 87, 489-499 (2002)

2. E.H. Hoogendoorn, A.R. Hermus, F. De VegT, H.A. Ross, A.L. Verbeek, L.A. Kiemeney, D.W. Swinkels, F.C. Sweep, M. den Heijer, Thyroid function and prevalence of anti-thyroperoxidase antibodies in a population with borderline sufficient iodine intake: influences of age and sex. Clin. Chem. 52, 104-111 (2006)

3. L.J. van Tienhoven-Wind, R.P. Dullaart, Low-normal thyroid function and the pathogenesis of common cardio-metabolic disorders. Eur. J. Clin. Investig. 45, 494-503 (2015)

4. P.N. Taylor, D. Albrecht, A. Scholz, G. Gutierrez-Buey, J.H. Lazarus, C.M. Dayan, O.E. Okosieme, Global epidemiology of hyperthyroidism and hypothyroidism. Nat. Rev. Endocrinol. 14, 301-316 (2018)

5. B.O. Åsvold, L.J. Vatten, T. Bjøro, D.C. Bauer, A. Bremner, A.R. Cappola, G. Ceresini, W.P. Den Elzen, L. Ferrucci, O.H. Franco, Thyroid function within the normal range and risk of coronary heart disease: an individual participant data analysis of 14 cohorts. JAMA Intern. Med. 175, 1037-1047 (2015)

6. R.P. Dullaart, R. De Vries, C. Roozendaal, A.C.M. Kobold, W.J. Sluiter, Carotid artery intima media thickness is inversely related to serum free thyroxine in euthyroid subjects. Clin. Endocrinol. 67, 668-673 (2007)

7. A. Bano, K. Dhana, L. Chaker, M. Kavousi, M.A. Ikram, F.U. Mattace-Raso, R.P. Peeters, O.H. Franco, Association of thyroid function with life expectancy with and without cardiovascular disease: the Rotterdam study. JAMA Intern. Med. 177, 1650-1657 (2017)
8. L.J.N. van Tienhoven-Wind, E.G. Gruppen, W.J. Sluiter, S.J.L. Bakker, R.P.F. Dullaart, Life expectancy is unaffected by thyroid function parameters in euthyroid subjects: the PREVEND cohort study. Eur. J. Intern. Med. 46, e36-e39 (2017)

9. J.B. Bertelsen, L. HEGEDÜS, Cigarette smoking and the thyroid. Thyroid 4, 327-331 (1994)

10. W.M. Wiersinga, Smoking and thyroid. Clin. Endocrinol. 79, 145-151 (2013)

11. A. Melander, E. Nordenskjöld, B. Lundh, J. Thorell, Influence of smoking on thyroid activity. Acta Med. Scand. 209, 41-43 (1981)

12. I. Meral, A. Arslan, A. Him, H. Arslan, Smoking-related alterations in serum levels of thyroid hormones and insulin in female and male students. Altern. Ther. Health Med. 21, 24-29 (2015)

13. R. Jorde, J. Sundsfjord, Serum TSH levels in smokers and nonsmokers. The 5th Troms $\varnothing$ study. Exp. Clin. Endocrinol. Diabetes 114, 343-347 (2006)

14. O.P. Soldin, B.E. Goughenour, S.Z. Gilbert, H.J. Landy, S.J. Soldin, Thyroid hormone levels associated with active and passive cigarette smoking. Thyroid 19, 817-823 (2009)

15. S. Kim, M.J. Kim, S.G. Yoon, J.P. Myong, H.W. Yu, Y.J. Chai, J. Y. Choi, K.E. Lee, Impact of smoking on thyroid gland: doserelated effect of urinary cotinine levels on thyroid function and thyroid autoimmunity. Sci. Rep. 9, 4213 (2019)

16. P. Valeix, P. Faure, S. Bertrais, A. Vergnaud, L. Dauchet, S. Hercberg, Effects of light to moderate alcohol consumption on thyroid volume and thyroid function. Clin. Endocrinol. 68, 988-995 (2008)

17. S. Ozsoy, E. Esel, H.B. Izgi, S. Sofuoglu, Thyroid function in early and late alcohol withdrawal: relationship with aggression, family history, and onset age of alcoholism. Alcohol Alcohol 41, 515-521 (2006)

18. S.N. Slagter, J.V. van Vliet-Ostaptchouk, J.M. Vonk, H.M. Boezen, R.P. Dullaart, A.C.M. Kobold, E.J. Feskens, A.P. van Beek, K. van der, M. Melanie, B.H: Wolffenbuttel, Combined effects of smoking and alcohol on metabolic syndrome: the LifeLines cohort study. PLoS ONE 9, e96406 (2014)

19. E.G. Gruppen, S.J. Bakker, R.W. James, R.P. Dullaart, Serum paraoxonase-1 activity is associated with light to moderate alcohol consumption: the PREVEND cohort study. Am. J. Clin. Nutr. 108, 1283-1290 (2018)

20. S.E. Borggreve, H.L. Hillege, B.H. Wolffenbuttel, P.E. de Jong, S.J. Bakker, G. van der Steege, A. van Tol, R.P. DullaartPREVEND Study Group, The effect of cholesteryl ester transfer protein $-629 \mathrm{C}->\mathrm{A}$ promoter polymorphism on high-density lipoprotein cholesterol is dependent on serum triglycerides. J. Clin. Endocrinol. Metab. 90, 4198-4204 (2005).

21. H.J. Lambers Heerspink, A.H. Brantsma, D. de Zeeuw, S.J. Bakker, P.E. de Jong, R.T. Gansevoort, PREVEND Study Group, Albuminuria assessed from first-morning-void urine samples versus 24-hour urine collections as a predictor of cardiovascular morbidity and mortality. Am. J. Epidemiol. 168, 897-905 (2008)

22. S.H. Koning, R.T. Gansevoort, K.J. Mukamal, E.B. Rimm, S.J. Bakker, M.M. Joosten, Alcohol consumption is inversely associated with the risk of developing chronic kidney disease. Kidney Int. 87, 1009-1016 (2015)

23. A. Abbasi, E. Corpeleijn, R.T. Gansevoort, R.O. Gans, H.L. Hillege, R.P. Stolk, G. Navis, S.J. Bakker, R.P. Dullaart, Role of HDL cholesterol and estimates of HDL particle composition in future development of type 2 diabetes in the general population: the PREVEND study. J. Clin. Endocrinol. Metab. 98, E1352-E1359 (2013)

24. R. Sediq, J. van der Schans, A. Dotinga, R.A. Alingh, B. Wilffert, J.H. Bos, C.C. Schuiling-Veninga, E. Hak, Concordance assessment of self-reported medication use in the netherlands threegeneration lifelines Cohort study with the pharmacy database 
iaDB. nl: the Pharmlines initiative. Clin. Epidemiol. 10, 981-989 (2018)

25. L.A. Inker, C.H. Schmid, H. Tighiouart, J.H. Eckfeldt, H.I. Feldman, T. Greene, J.W. Kusek, J. Manzi, F. Van Lente, Y.L. Zhang, Estimating glomerular filtration rate from serum creatinine and cystatin C. N. Engl. J. Med. 367, 20-29 (2012)

26. S.P. Matyus, P.J. Braun, J. Wolak-Dinsmore, E.J. Jeyarajah, I. Shalaurova, Y. Xu, S.M. Warner, T.S. Clement, M.A. Connelly, T.J. Fischer, NMR measurement of LDL particle number using the Vantera clinical analyzer. Clin. Biochem. 47, 203-210 (2014)

27. J. Kang, E. Kong, J. Choi, Associations of urinary cotinineverified active and passive smoking with thyroid function: analysis of population-based nationally representative data. Thyroid 28, 583-592 (2018)

28. R.M. Belin, B.C. Astor, N.R. Powe, P.W. Ladenson, Smoke exposure is associated with a lower prevalence of serum thyroid autoantibodies and thyrotropin concentration elevation and a higher prevalence of mild thyrotropin concentration suppression in the third National Health and Nutrition Examination Survey (NHANES III). J. Clin. Endocrinol. Metab. 89, 6077-6086 (2004)

29. P.E. Cryer, M.W. Haymond, J.V. Santiago, S.D. Shah, Norepinephrine and epinephrine release and adrenergic mediation of smoking-associated hemodynamic and metabolic events. N. Engl. J. Med. 295, 573-577 (1976)

30. L. Mehran, A. Amouzgar, H. Delshad, F. Azizi, The association of cigarette smoking with serum TSH concentration and thyroperoxidase antibody. Exp. Clin. Endocrinol. Diabetes 120, 80-83 (2012)

31. S. Park, W.G. Kim, M.J. Jeon, M. Kim, H. Oh, M. Han, T.Y. Kim, Y.K. Shong, W.B. Kim, Serum thyroid-stimulating hormone levels and smoking status: data from the Korean National Health and Nutrition Examination Survey VI. Clin. Endocrinol 88, 969-976 (2018)
32. R.P. Dullaart, K. Hoogenberg, B.D. Dikkeschei, A. van Tol, Higher plasma lipid transfer protein activities and unfavorable lipoprotein changes in cigarette-smoking men. Arterioscler. Thromb. 14, 1581-1585 (1994)

33. L. Chaker, C. Baumgartner, W.P. Den Elzen, T. Collet, M.A. Ikram, M.R. Blum, A. Dehghan, C. Drechsler, R.N. Luben, M.L. Portegies, Thyroid function within the reference range and the risk of stroke: an individual participant data analysis. J. Clin. Endocrinol. Metab. 101, 4270-4282 (2016)

34. V. van de, C. Annenienke, R.T. Netea-Maier, F. de Vegt, H.A. Ross, F.C. Sweep, L.A. Kiemeney, J.W. Smit, A.R. Hermus, M. den Heijer, Associations between thyroid function and mortality: the influence of age. Eur. J. Endocrinol. 171, 183-191 (2014)

35. V. van de, C. Annenienke, R.T. Netea-Maier, J.W. Smit, R. Kusters, S. van der, W.J. Jos, C.J. Pronk-Admiraal, M.M. Buijs, C.H. Schoenmakers, S.G. Koehorst, M.J. de Groot, Thyrotropin versus age relation as an indicator of historical iodine intake. Thyroid 25, 629-634 (2015)

36. M. Geurts, J. Verkaik-Kloosterman. De jodiuminname van de Nederlandse bevolking na verdere zoutverlaging in brood. RIVM. (2014)

37. H.G. Kang, K.H. Kwon, I.W. Lee, B. Jung, E. Park, S. Jang, Biochemically-verified smoking rate trends and factors associated with inaccurate self-reporting of smoking habits in Korean women. Asian Pac. J. Cancer Prev. 14, 6807-6812 (2013)

38. S.K. Kunutsor, J.M. Spee, L.M. Kieneker, R.T. Gansevoort, R.P. Dullaart, A. Voerman, D.J. Touw, S.J. Bakker, Self-Reported smoking, urine cotinine, and risk of cardiovascular disease: findings from the PREVEND (Prevention of Renal and Vascular EndStage Disease) prospective cohort study. J. Am. Heart Assoc. 7, e008726 (2018) 